\title{
In-fiber reflection mode interferometer based on a long-period grating for external refractive-index measurement
}

\author{
Dae Woong Kim, Yan Zhang, Kristie L. Cooper, and Anbo Wang
}

\begin{abstract}
We present two novel schemes for refractometry based on a long-period fiber grating- (LPG-) based Michelson interferometer. These schemes are designed to overcome the measurement dependence of previously demonstrated LPG-based refractometry on the immersion depth. The first utilizes an unshielded LPG and the second, a shielded one. Both schemes were tested over a certain refractive-index range, and the measurement of glucose concentration in water was experimentally demonstrated. In addition, the temperature sensitivity of the two schemes is discussed. (c) 2005 Optical Society of America OCIS codes: $350.2770,060.2370,060.2340$.
\end{abstract}

\section{Introduction}

The development of devices for refractometry has become increasingly important for applications in industrial process and quality control, biomedical analysis, and environmental monitoring. Various types of optical fiber refractometer that employ long-period gratings $^{1-3}$ (LPGs), etched fiber Bragg gratings, ${ }^{4,5}$ a tilted fiber Bragg grating, ${ }^{6}$ a surface plasmon resonance sensing structure, ${ }^{7}$ and a glass hemisphere sensing element ${ }^{8}$ have been widely investigated for these applications. Among these, greater attention has been paid to sensors based on LPGs because of their high sensitivity and relatively simple fabrication and signal detection.

A pair of identical long-period gratings written in a photosensitive fiber exhibits a fringe pattern in each resonance spectral band. ${ }^{9}$ The first LPG couples light from the core mode to a cladding mode. The coupled cladding mode propagates in the cladding and is recoupled to the core mode by the second LPG, while the uncoupled core mode continues to propagate through the core region. Light from these two optical paths interferes through the second LPG, functioning

The authors are with the Center for Photonics Technology, Bradley Department of Electrical and Computer Engineering, Virginia Polytechnic Institute and State University, Blacksburg, Virginia 24061-0111. D. W. Kim's e-mail address is dakim1@vt.edu.

Received 4 January 2005; revised manuscript received 21 April 2005; accepted 16 May 2005.

$0003-6935 / 05 / 265368-06 \$ 15.00 / 0$

(C) 2005 Optical Society of America as an in-fiber Mach-Zehnder interferometer. Because this configuration has better resolution and higher sensitivity than the conventional single LPGbased sensors in which only core-to-cladding coupling wavelengths are measured, devices based on this scheme have been developed for use as optical sensors $^{10}$ and in-fiber components for optical communication systems. ${ }^{11}$ An interferometer operating in reflection mode has been demonstrated by formation of a mirror at the end of a fiber containing a LPG for temperature $^{12}$ and refractive-index ${ }^{13}(\mathrm{RI})$ sensing. This simple structure has the same spectral properties as a LPG pair but offers advantages such as compact size and the ability to use a single-ended probe in some applications. Because the temperature cross sensitivity of such a sensor is still a critical issue for refractometry, several approaches have been proposed to overcome the cross sensitivity. For example, a sensor that uses two types of optical fiber with opposing thermal properties was recently demonstrated. ${ }^{14}$ It has also been suggested ${ }^{15}$ that the envelope of the interference fringes could be used for temperature measurement if a LPG were shielded from the target medium, but to the best of our knowledge this has yet to be demonstrated. In addition, the refractometry schemes ${ }^{13-15}$ result in a sensitivity that is dependent on the interaction length of the sensor, i.e., on its depth of immersion into the medium whose index is being measured. Devising a new scheme that overcomes this cross sensitivity is imperative for practical applications.

In this paper we report two novel schemes, which use total immersion and partial immersion of the 


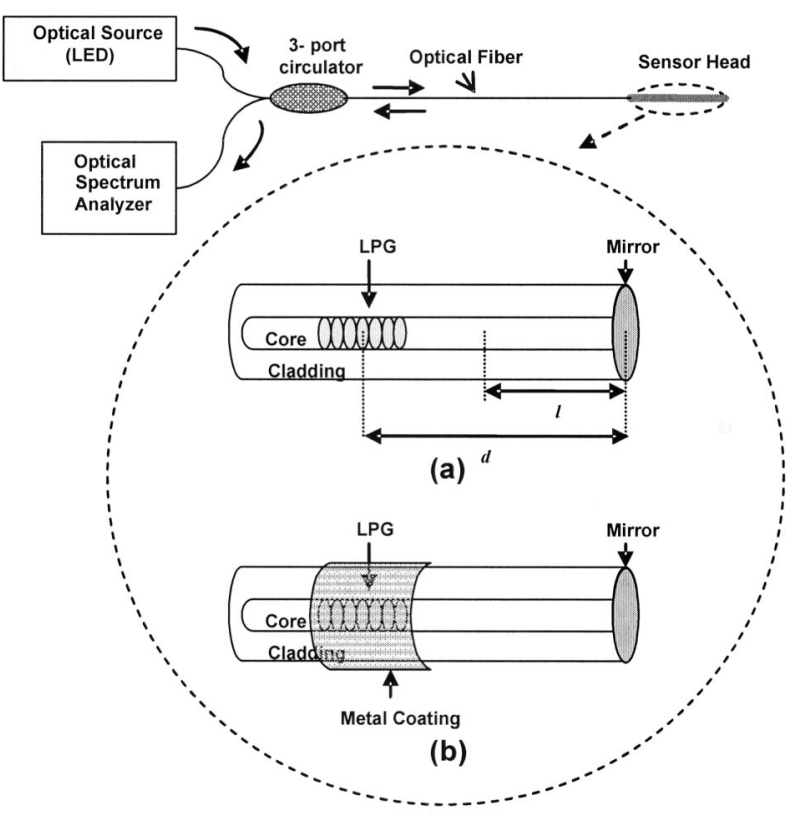

Fig. 1. Experimental setup and schematics of the proposed sensors using (a) total and (b) partial immersion of the sensitive area. The total immersion scheme requires that $l>d+$ half the total grating length; partial immersion requires that $l$ be greater than the distance from the metal coating to the fiber end.

sensor, that make the sensor easier to use in practical measurement. Using both schemes, we investigated the spectral response of the sensors to the surrounding $R I$ for $R I$ values below the cladding RI, and we experimentally demonstrated their practical feasibility by measuring the glucose concentration in water over a range of $0 \%-30 \%$. The results show that the proposed sensing schemes have high sensitivity and stability. In addition, the cross sensitivity of the sensors to temperature was measured and is discussed.

\section{Operating Principle and Fabrication Process}

Diagrams of the proposed sensors and their experimental configuration are shown in Fig. 1. Light propagating in the core is divided into two paths by a LPG with 50\% coupling strength, forming the two arms of a Michelson interferometer. The light in each path is reflected by a mirror fabricated directly on the fiber end and then recombined into the core by the same LPG, giving rise to interference fringes in each resonance band. The wavelength at which the copropagating coupling occurs is given by ${ }^{16}$

$$
\lambda_{i}=\left(n_{\mathrm{co}}-n_{\mathrm{cl}, i}\right) \Lambda,
$$

where $\lambda_{i}$ is the resonance wavelength that corresponds to the $i$ th cladding mode $\left(\mathrm{HE}_{1 i}\right), n_{\mathrm{co}}$ and $n_{\mathrm{cl}, i}$ denote the effective indices of the guided mode $\left(\mathrm{HE}_{11}\right)$ and the $i$ th cladding mode, respectively, and $\Lambda$ is the grating period. If any perturbation that changes the phase difference is applied to one arm of the interferometer, i.e., the optical path through the cladding, the interference pattern will be modulated. The phase difference in the two interferometer arms can be expressed as

$$
\Delta \phi=\frac{4 \pi}{\lambda}\left[\left(n_{\mathrm{co}}-n_{\mathrm{cl}, i}\right) d+\Delta n_{\mathrm{cl}, i} l\right]
$$

where $\Delta n_{\mathrm{cl}, i}$ is the change in the effective RI of the $i$ th cladding mode caused by the immersion of the sensor into the testing medium, $d$ is the distance between the grating center and the reflector, and $l$ is the fiber immersion length (interaction length). Equation (2) implies the dependence of the RI-induced fringe shift on the cladding mode and the interaction length of the sensor. The fringe shift caused by a surrounding RI change thus increases with an increase in the interaction length, which means that the sensor requires a fixed immersion depth for RI measurement, making it difficult to use this sensor practically. To overcome this cross sensitivity, two schemes that use total or partial immersion of the sensor have been proposed and applied as follows.

To demonstrate the two sensors, we inscribed a LPG with an $\mathrm{HE}_{18}$ cladding mode and a resonance wavelength of approximately $1.56 \mu \mathrm{m}$ in a photosensitive fiber (Thorlabs, GF1) using a point-by-point technique. The UV light launched by a cw frequencydoubled argon laser was focused onto the photosensitive fiber via a focal lens, and the fiber was then translated by use of a computer-controlled step motor. The attenuation intensity of the desired cladding mode was adjusted to have $\sim 3 \mathrm{~dB}$ coupling strength. The LPG had a grating period of $310 \mu \mathrm{m}$ and a total grating length of $\sim 17 \mathrm{~mm}$. The end of the fiber containing the LPG was cleaved by a high-precision fiber cleaver, inserted into a tube with an inner diameter of $130 \mu \mathrm{m}$ to prevent coating on the sensing region, and then coated with silver by a silver mirror reaction (Tollen's test). For the total immersion scheme, shown in Fig. 1(a), the tubing covered the entire sensing region of the sensor, forming a mirror on the end surface of the fiber; for the scheme that used partial immersion, shown in Fig 1(b), the tubing covered only the grating-free region of the sensor, forming a silver film on the surface of the grating region and also at the end of the fiber. A silver nitrate solution $(2 \mathrm{ml}, 0.1 \mathrm{M})$ was placed in a tube. Ammonium hydroxide was added to the solution drop by drop, with stirring, until the brown precipitate just dissolved. A potassium hydroxide solution $(1.4 \mathrm{ml}, 0.8 \mathrm{M})$ was then added. If the brown precipitate re-formed, additional ammonium hydroxide was added until it dissolved. Then the Tollen's reagent was ready for use. The sensor was rinsed with a stannous chloride solution $(0.2 \%)$ to sensitize the coating area and then rinsed with de-ionized water. A dextrose solution $(0.4 \mathrm{ml}, 0.25 \mathrm{M})$ was poured into the tube, and the fiber sensor with the tubing was then dipped into the Tollen's reagent. The silver film formed within $\sim 1 \mathrm{~min}$. Finally, the fiber was thoroughly cleaned with de-ionized water. It was observed that a uniform coating formed on the surface of the LPG and a highquality film formed on the end surface of the fiber. 
The silver film's thickness was estimated to be more than $200 \mathrm{~nm}$. Because the quality of the reflection surface is a critical factor in determining the contrast of the interference pattern, we examined each sensor before we coated it with silver to select those that had good fringe contrast. The distance between the grating and the mirror is $14 \mathrm{~mm}$, and the total sensor length with the grating included is $\sim 31 \mathrm{~mm}$. Interference fringe patterns in the reflection spectrum were measured by a broadband light-emitting diode (LED) at $1.55 \mu \mathrm{m}$ and an optical spectrum analyzer with $0.1 \mathrm{~nm}$ resolution, which was connected to a computer for signal processing. All subsequent spectra shown in this paper were normalized with the measured LED spectrum, and the data from the optical spectrum analyzer were analyzed with a Matlab program to locate the fringe positions. A circulator was used to separate the input and output light of the sensor. All the sensors were annealed at $110{ }^{\circ} \mathrm{C}$ for $24 \mathrm{~h}$ to stabilize their spectral response before the test, and a small shift $(\sim 1 \mathrm{~nm})$ of the fringe positions to shorter wavelengths was observed after the annealing.

\section{Experimental Results}

\section{A. Sensing Scheme Using Total Immersion}

For the measurement of an external RI, the entire sensor including the LPG was immersed in liquids with different refractive indices at room temperature. The difference between this scheme and those reported earlier ${ }^{13-15}$ is in the immersion of the entire sensor, which allows us to use the scheme without additional effort to fix the sensor at a certain immersed depth for each measurement.

Figure 2(a) shows the interference fringes in the eighth cladding mode resonance band and the RIinduced fringe shift in the reflection spectrum. An increase in RI shifted both the resonance band and the fringes within the resonance band toward shorter wavelengths compared with that of air. Figure 2(b) presents the shift of the fringe position that is due to the external RI change. The fringe with the deepest attenuation was traced as the external RI was increased from 1.000 to 1.452 . As the surrounding index increased, all the fringes shifted to shorter wavelengths, and the visibility deteriorated. When the ambient index was close to the cladding's refractive index $(\sim 1.455)$, the interference pattern disappeared because discrete cladding modes no longer existed.

To explore the performance of this sensor for practical applications, we used the sensor to measure the concentration of glucose solutions whose refractive index is already known. Changes in glucose concentration from $0 \%$ to $30 \%$ (mass \%) correspond to changes in RI from 1.333 to 1.381 at a wavelength of $589 \mathrm{~nm} .{ }^{17}$ The position of the deepest fringe was traced for concentration steps of $2 \%$, which correspond to a RI change of approximately $3.2 \times 10^{-3}$. The experimental results are shown in Fig. 3: A second-order polynomial curve fit was applied to the average values of

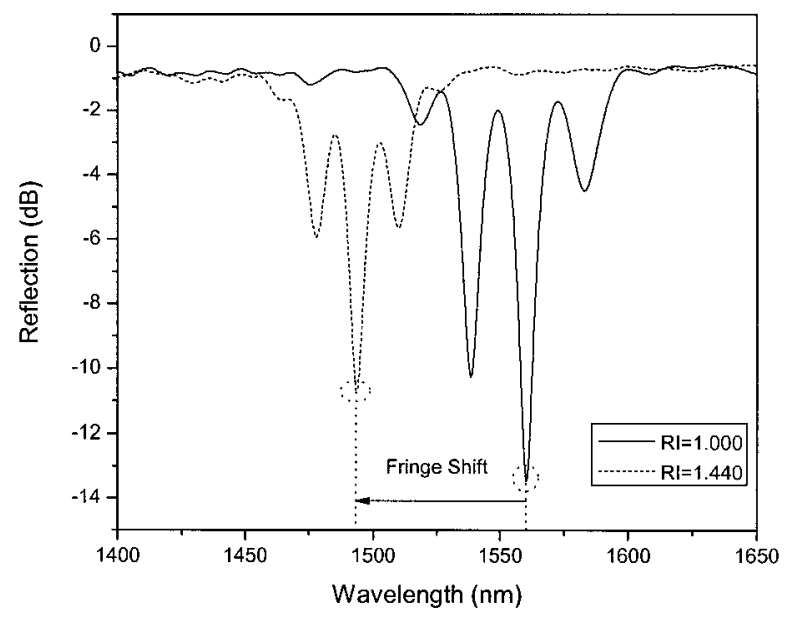

(a)

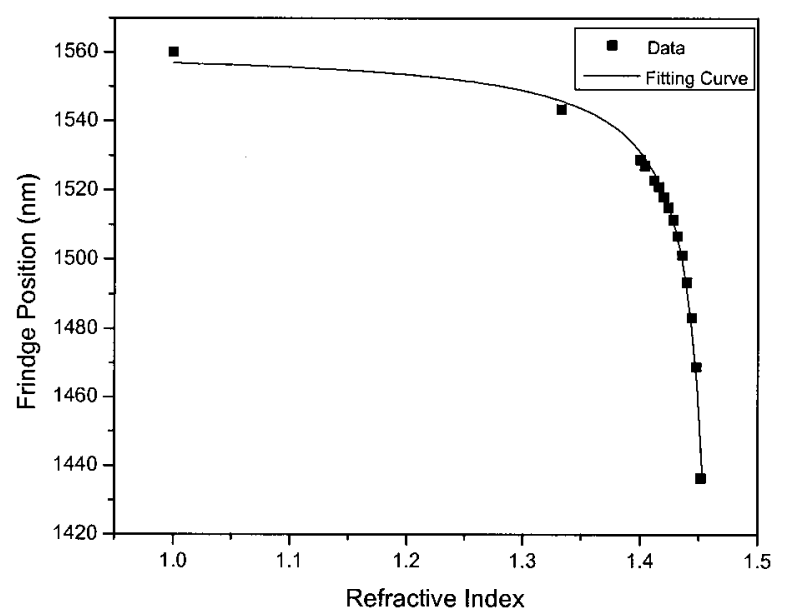

(b)

Fig. 2. Shifts in interference fringe owing to changes in the surrounding RI. The entire sensor including the LPG was immersed. (a) Interference fringes in a $\mathrm{HE}_{18}$ cladding mode resonance band and its fringe shift, (b) dependence of the wavelength shift of fringe position on the surrounding RI.

the data points obtained from multiple measurements. The sensor was cleaned with de-ionized water and acetone after each measurement. The result shows that this sensor is sensitive to an external RI change of the order of $10^{-4}$ in this RI range and can thus be used for measurement of concentrations of various chemicals.

\section{B. Sensing Scheme Using Partial Immersion}

For the second approach, the LPG portion of the sensor used for the first scheme was coated with silver to shield the electromagnetic field of the cladding mode in the metal-coated region, as shown in Fig. 1(b). Because only a small portion of the evanescent field of a cladding mode penetrates deeply into a metal, 18 the metal film can protect the LPG region from the influence of the surrounding medium. It is thus expected that the resonance band envelope will be fixed and the interference fringes within the band will change with the external RI. To verify the protection 


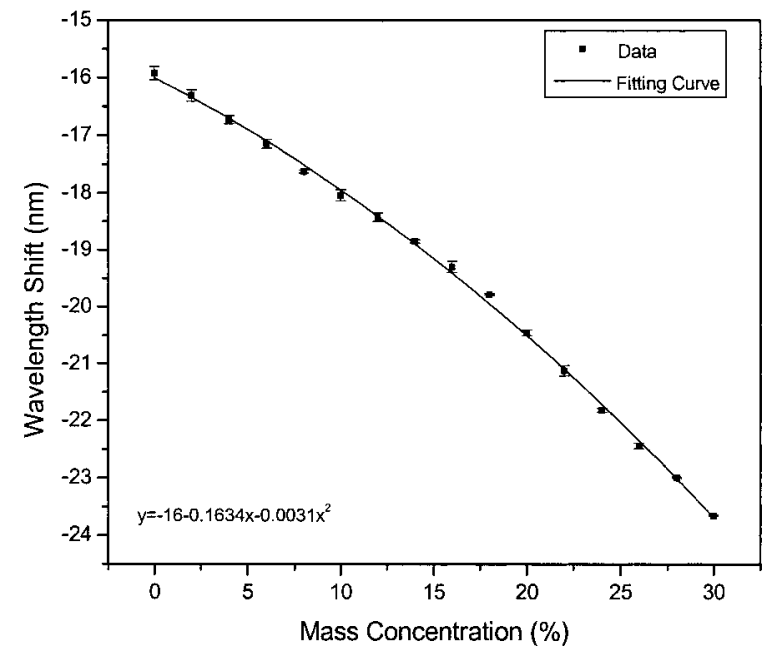

Fig. 3. Measurement of glucose concentration by the total immersion scheme.

of the LPG portion from the surrounding RI change, we immersed the entire sensor probe into liquids with various RIs. As expected, no spectral shift of the resonance band was observed. The presence of the metal film on the LPG caused spectral changes of the original resonance band, such as in coupling strength, center wavelength, and bandwidth. Figure 4 shows the reflection spectra before and after deposition of the metal coating onto the LPG region. The visibility of the sensor was degraded and a fringe shift of approximately $40 \mathrm{~nm}$ to longer wavelengths was observed after the metal coating. Figure 5(a) shows the reflection spectrum of the sensor and the fringe shift caused by the RI change. In this case the resonance band did not shift but the fringe positions shifted to shorter wavelengths. The position of the rightmost fringe in the figure was traced for measurement over a RI range of 1.000 to 1.436 because it provides a larger displacement than the remaining two fringes. The results are shown in Fig. 5(b). RIs exceeding

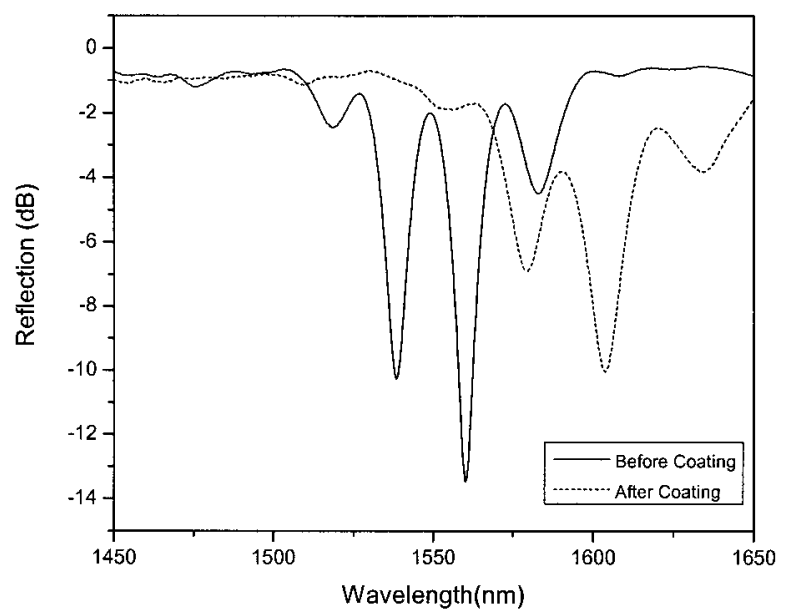

Fig. 4. Reflection spectra of the sensor before and after deposition of a metal coating upon the LPG area.

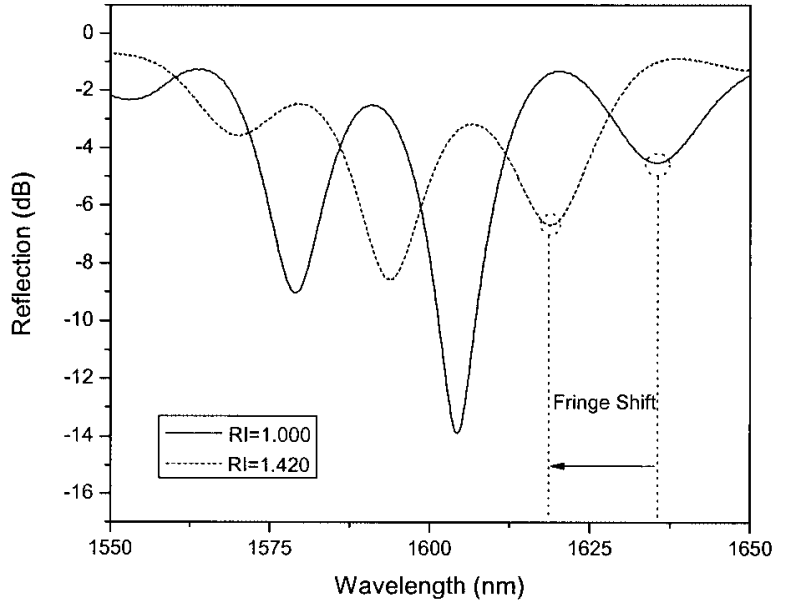

(a)

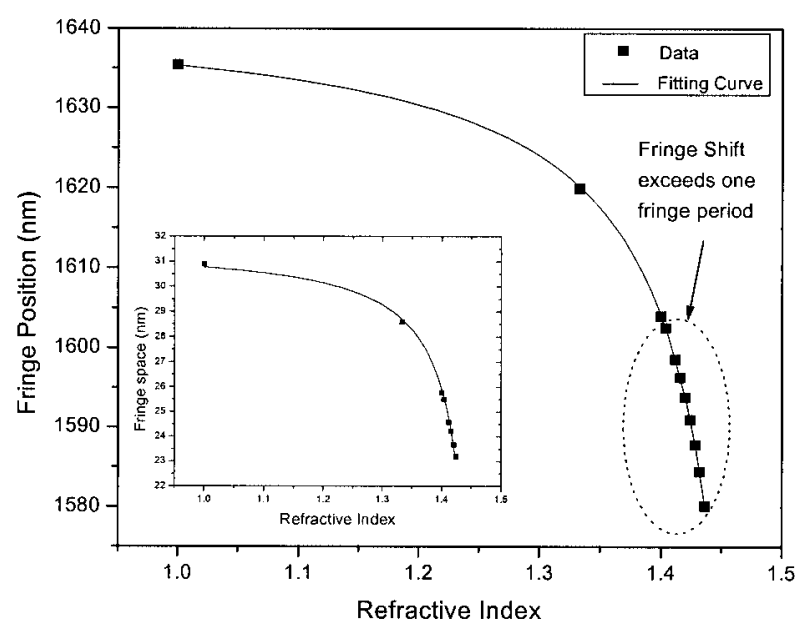

(b)

Fig. 5. Interference fringe shifts that are due to changes in the surrounding RI. The grating-free region of the sensitive area was used. (a) Interference fringes in a $\mathrm{HE}_{18}$ cladding mode resonance band and its fringe shift, (b) dependence of the wavelength shift of the fringe and (inset) the average spacing of the fringes on the surrounding RI.

1.404 resulted in a phase ambiguity problem. When the fringe shift exceeds one fringe period, the traced fringe cannot be identified. To overcome this limitation we calculated the average spacing of the fringe valley points, which is shown in the inset of Fig. 5(b). The fringe spacing decreased as the external RI increased. The calculation was carried out over a RI range of 1.00 to 1.424 , as the contrast was insufficient to allow the fringe positions to be traced above that value. The results show that we can use this information as a reference signal to identify the traced fringe. In comparison to the first approach, which uses the entire sensitive region, this scheme has less sensitivity and a narrower measurement range owing to the poor contrast. However, it permits the application of white-light interferometric signal demodulation $^{19}$ for automated absolute measurement because of the fixed resonance band's spectral profile. 


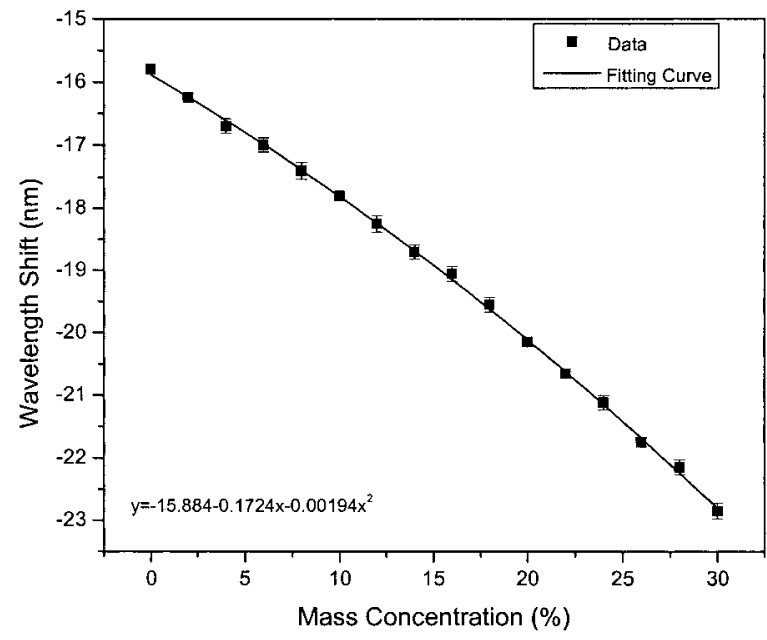

Fig. 6. Measurement of glucose concentration by the partial immersion scheme.

Further, white-light signal processing permits simple demodulation of multiple interferometers with different differential optical path lengths, ${ }^{20}$ so an additional Fabry-Perot interferometric temperature sensor can be placed in close proximity to the LPG sensor for temperature compensation. These enhancements are beyond the scope of this paper.

The glucose concentration measurement was also conducted with this new structure. Figure 6 shows the shift of the fringe that is due to a change in the mass concentration of glucose. It shows a sensitivity that is similar to that of the first scheme and does not exhibit the phase ambiguity problem in this RI range.

\section{Discussion}

Figure 7 shows the thermally induced fringe shift of the sensor. The sensor was placed in an oven, and the reflection spectra were measured while the oven temperature was varied from $20.0{ }^{\circ} \mathrm{C}$ to $80.0{ }^{\circ} \mathrm{C}$. The fringe with the deepest attenuation was traced, and a linear fit to the experimental data has a slope of approximately $-0.098 \mathrm{~nm} /{ }^{\circ} \mathrm{C}$ for both schemes. The difference between the temperature sensitivities of the sensors for the two different schemes was barely distinguishable in this experiment. As the temperature of the environment or the medium that we desire to measure may not always be stable, the spectral drift of the sensor caused by temperature change should be minimized. The thermally induced fringe shift of the sensor can be approximated as

$$
\frac{\partial \lambda}{\partial T}=\left(\frac{\partial \Delta n_{\mathrm{eff}, i}}{\partial T}+\alpha \Delta n_{\mathrm{eff}, i}\right) \Lambda
$$

where $\Delta n_{\text {eff }, i}$ is the differential effective RI of the core and the $i$ th cladding mode and $\alpha$ is the thermal expansion coefficient of fused silica $\left(0.55 \times 10^{-6} /{ }^{\circ} \mathrm{C}\right)$. The first term describes the dependence of the effective indices on temperature, and the second term describes the thermal expansion of the fiber. It fol-

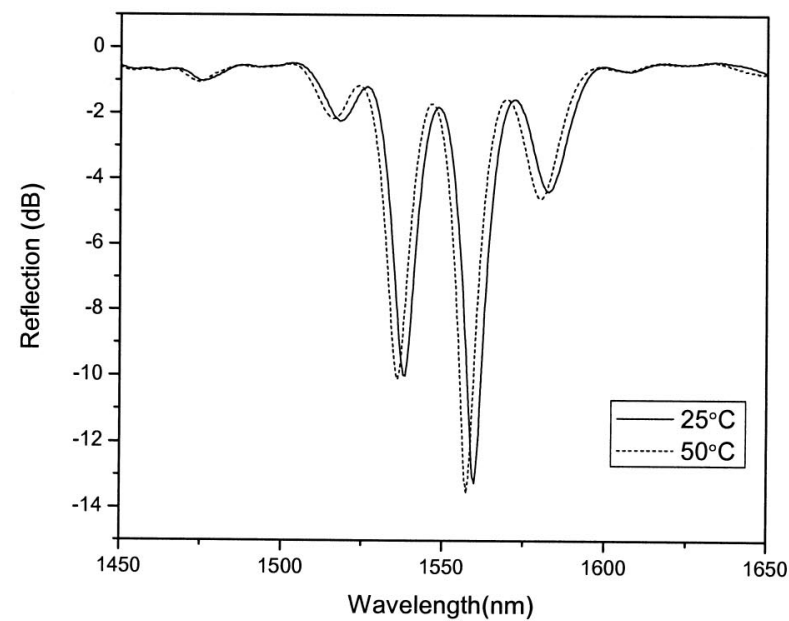

(a)

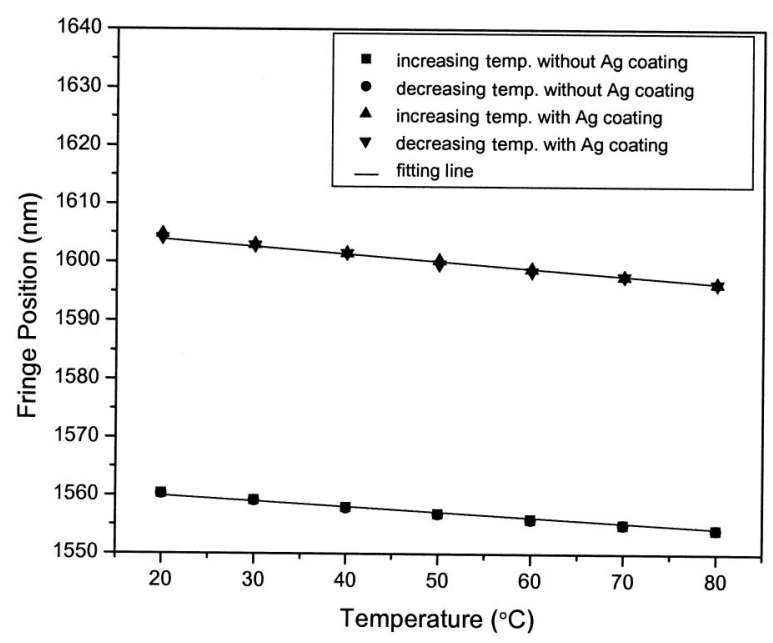

(b)

Fig. 7. Thermally induced interference fringe shift: (a) reflection spectra of the sensor without a metal coating on the LPG and its fringe shift, (b) dependence of the fringe shift on temperature.

lows that the temperature sensitivity of the sensor is dependent on the cladding mode order and also varies with different types of fiber. The temperature sensitivity of the sensor can thus be decreased by choice of a photosensitive fiber or by direct adjustment of fiber dopants for lower sensitivity. Another approach is to employ a (short-period) fiber Bragg grating or an intrinsic Fabry-Perot interferometer, which are insensitive to the external RI, for temperature sensing and then to compensate for the thermally induced drift of the output signal.

The experimental results show that the schemes that use partial or total immersion of the sensor can both be used for external RI measurement. The tip of the sensor probe was placed downward to prevent or minimize undesirable bending and vibrations from the environment, and the probe was immersed to a random depth exceeding the interferometric cavity length. Therefore the sensor is especially suitable for applications in which it must be immersed in the 
target medium. The sensitivity is dependent on the cladding mode order, similarly to the spectral behavior of a normal LPG. The sensitivity can be increased by choice of an appropriate (higher-) order cladding mode or etching of the cladding of the sensitive part of the sensor to reduce the diameter.

To show the stability and repeatability of the proposed sensors, we measured the glucose concentration with the sensors, showing that these sensors can be used for various chemical (gas) and biological analyses. As one of the interesting applications, species-specific chemical or biological sensors can be fabricated with these sensing schemes by deposition of a thin film that changes its RI in response to a target on the sensitive part of the sensors.

\section{Conclusions}

In this paper we have presented in-fiber interferometers that include a long-period grating operating in reflection mode for refractive-index sensing. To overcome the immersion-length dependence limitation of the reflection mode LPG sensor reported earlier, we have proposed and demonstrated two novel sensing schemes, including a new design for the sensor probe. The sensors for both the schemes exhibit RI sensitivity of the order of $10^{-4}$ over the range of 1.333-1.38. This device offers the advantages of small size, ease of use, high sensitivity, and new functionality, offering potential for various applications in the future.

\section{References}

1. V. Bhatia and A. M. Vengsarkar, "Optical fiber long-period grating sensors,” Opt. Lett. 21, 692-694 (1996).

2. L. S. Thomas, N. A. George, P. Sureshkumar, P. Radhakrishnan, C. P. G. Vallanbhan, and V. P. N. Namppori, "Chemical sensing with microbent optical fiber," Opt. Lett. 26, 1541-1543 (2001).

3. N. D. Rees, S. W. James, R. Tatam, and G. J. Ashwell, "Optical fiber long-period gratings with Langmuir-Blodgett thin-film overlays," Opt. Lett. 27, 686-688 (2002).

4. A. Asseh, S. Sandgren, H. Ahlfeldt, B. Sahlgren, R. Stubbe, and G. Edwall, "Fiber optical Bragg grating refractometer," Fiber Integr. Opt. 17, 51-62 (1998).

5. K. Shroeder, W. Ecke, R. Mueller, R. Willsch, and A. Andreev, "A fibre Bragg grating refractometer," Meas. Sci. Technol. 12, 757-764 (2001).
6. G. Laffont and P. Ferdinand, "Tilted short-period fibre-Bragggrating-induced coupling to cladding modes for accurate refractometry," Meas. Sci. Technol. 12, 765-770 (2001).

7. R. Slavik, J. Homola, J. Čtyroky, and E. Brynda, "Novel spectral fiber optic sensor based on surface plasmon resonance," Sens. Actuators B 74, 106-111 (2001).

8. V. Svirid, S. Khotiaintsev, and P. L. Swart, "Linear and steplike characteristics in an optical fiber refractometric transducer with hemispherical detection element," Opt. Eng. 42, 1383-1389 (2003).

9. E. M. Dianov, S. A. Vasiliev, A. S. Kurkov, O. I. Medvedkov, and V. N. Protopopov, "In fiber Mach-Zehnder interferometer based on a pair of long period gratings," in Proceedings of the 22nd European Conference on Optical Communication (Telenor R\&D, Oslo, Norway, 1996), pp. 65-68.

10. Y. G. Han, B. H. Lee, W. T. Han, U. C. Paek, and Y. J. Chung, "Fibre optic sensing applications of a pair of long period fibre gratings," Meas. Sci. Technol. 12, 778-781 (2001).

11. X. J. Gu, "Wavelength-division multiplexing isolation fiber filter and light source using cascaded long-period fiber gratings," Opt. Lett. 23, 509-510 (1998).

12. B. H. Lee and J. Nishii, "Self-interference of long-period fibre grating and its application as temperature sensor," Electron. Lett. 34, 2059-2060 (1998).

13. P. L. Swart, "A single fibre Michelson interferometric sensor," in Proceedings of the 16th International Conference on Optical Fiber Sensors, K. Hotate and H. Nagai, eds. (Institute of Electronics, Information, and Communication Engineers, Tokyo, Japan, 2003), pp. 602-605.

14. A. Van Brakel and P. L. Swart, "Temperature-compensated optical fiber Michelson refractometer," Opt. Eng. Lett. 44, 1576-1580 (2005).

15. P. L. Swart, "Long-period grating Michelson refractometric sensor," Meas. Sci. Technol. 15, 1576-1580 (2004).

16. A. M. Vengsarkar, P. J. Lemaire, J. B. Judkins, V. Bhatia, T. Erdogan, and J. E. Sipe, "Long-period fiber gratings as bandrejection filters," J. Lightwave Technol. 14, 58-64 (1996).

17. D. R. Lide, ed., CRC Handbook of Chemistry and Physics, 84th ed. (CRC Press, 2003).

18. A. Yariv and P. Yeh, Optical Waves in Crystals (WileyInterscience, 1984).

19. B. Qi, G. Pickrell, J. C. Xu, P. Zhang, Y. H. Duan, W. Peng, Z. Y. Huang, W. Huo, H. Xiao, R. G. May, and A. Wang, "Novel data processing techniques for dispersive white light interferometer," Opt. Eng. 42, 3165-3171 (2003).

20. F. Shen and A. Wang, "Frequency-estimation-based signal processing algorithm for white-light optical fiber Fabry-Perot interferometers," Appl. Opt. (to be published). 